\section{Prevalência e fatores associados ao tabagismo em fumicultores do Sul do Brasil}

\author{
Smoking prevalence and associated factors among \\ tobacco farmers in southern Brazil
}

\section{Prevalencia y factores asociados al tabaquismo en \\ cultivadores de tabaco del sur de Brasil}

\section{Resumo}

O objetivo foi descrever a prevalência de tabagismo e fatores associados em fumicultores. Estudo transversal de base populacional, em 2.464 fumicultores, em 2011. Amostra obtida por randomização das notas fiscais emitidas pelos trabalhadores. A análise multivariada em homens avaliou a associação entre tabagismo e variáveis socioeconômicas, comportamentais e ocupacionais; $31,2 \%$ dos homens e 3,1\% das mulheres eram tabagistas atuais. Entre homens, tabagismo associou-se diretamente à idade, escolaridade, renda, ser bebedor pesado, tempo de trabalho na fumicultura e tempo de exposição a pesticidas. Relação de trabalho foi um fator de risco para tabagismo e participar de atividades religiosas um fator de proteção. Fumicultores homens apresentaram múltiplos comportamentos de risco e uma prevalência de tabagismo maior do que outros agricultores. A naturalização do risco e a herança cultural podem ser fatores comuns a tais comportamentos e sugerem abordagens conjuntas.

Tabagismo; Tabaco; Agricultura; População Rural
Nadia Spada Fiori 1

Neice Muller Xavier Faria 2

Rodrigo Dalke Meucci 1

Anaclaudia Gastal Fassa 1
1 Faculdade de Medicina, Universidade Federal de Pelotas, Pelotas, Brasil. 2 Secretaria Municipal de Saúde de Bento Gonçalves, Bento Gonçalves, Brasil.

\author{
Correspondência \\ N. S. Fiori \\ Departamento de Medicina \\ Social, Faculdade de \\ Medicina, Universidade \\ Federal de Pelotas. \\ Rua Duque de Caxias 250, \\ Pelotas, RS 96030-000, Brasil. \\ nsfiori@yahoo.com.br
}




\section{Introdução}

O Brasil é reconhecido mundialmente pela abrangência do seu Programa Nacional para o Controle do Tabagismo e por ter coordenado o processo de elaboração da Convenção Quadro para o Controle do Tabaco (CQCT) entre 1999 e 2003 1. Apesar da ratificação da CQCT no país em 2005 e dos esforços bem-sucedidos para se reduzir a prevalência de fumantes e a consequente morbimortalidade relacionada ao tabaco, o país é atualmente o segundo maior produtor e maior exportador de tabaco do mundo.

O uso do tabaco é reconhecido globalmente como a principal causa mundial de morte evitável, sendo responsável por cerca de $6 \mathrm{mi}$ lhões de mortes e mais de meio trilhão de dólares de danos econômicos, no mundo, a cada ano 2,3. Levantamentos de base populacional sobre a prevalência de tabagismo têm ocorrido periodicamente nos 180 países signatários da CQCT 4 e mostram uma tendência de redução na prevalência de tabagismo, especialmente em países que obtiveram sucesso nas medidas de controle do tabaco, permanecendo ainda grande variabilidade nas proporções de consumo de tabaco no mundo 4,5,6. Segundo a Organização Mundial da Saúde (OMS), na grande maioria das regiões, inclusive no Brasil, homens de nível econômico mais baixo são mais propensos a ser tabagistas atuais ?.

Inquéritos populacionais em países desenvolvidos analisaram a prevalência de tabagismo em diferentes ocupações e encontraram maiores prevalências em trabalhadores manuais 8,9 . Da mesma forma, a Pesquisa Nacional por Amostra de Domicílios de 2008 (PNAD 2008) constatou que trabalhadores de cargos com menor exigência de escolaridade e maior esforço braçal fumavam mais e que trabalhadores agrícolas tinham um consumo de cigarros duas vezes maior (21,9\%) que profissionais das áreas de ciências e artes 10. Embora os estudos em países desenvolvidos considerem o efeito da ocupação na prevalência de tabagismo decorrente do nível socioeconômico 8,9, pois trabalhadores manuais têm menor escolaridade e renda, o estudo brasileiro reforça o papel da ocupação na determinação do tabagismo 10 .

Estudo em fumicultores indianos encontrou uma prevalência de tabagismo em homens de $68,7 \%$, sugerindo que os trabalhadores da fumicultura possam ter prevalências mais altas que outros agricultores e trabalhadores urbanos 11 . Assim, considerando a abrangência do programa brasileiro de controle do tabagismo, a magnitude da fumicultura brasileira, que envolve mais de 220 mil famílias (Instituto Brasileiro de Geografia e Estatística. http://www.ibge.gov.br/cidadesat, acessado em Mai/2015), e de não haver estudos sobre tabagismo nesse grupo de trabalhadores brasileiros, o presente estudo objetiva descrever a prevalência de tabagismo e seus fatores associados em fumicultores do Sul do Brasil.

\section{Métodos}

Conduziu-se um estudo transversal com amostragem aleatória de fumicultores do Município de São Lourenço do Sul, Estado do Rio Grande do Sul, Brasil, no período da colheita do ano de 2011 (janeiro a março).

São Lourenço do Sul está situado ao sul do estado, com 43.111 habitantes e índice de desenvolvimento humano do município (IDH-M) de 0,687 (Instituto Brasileiro de Geografia e Estatística. http://www.ibge.gov.br/cidadesat, acessado em Mai/2015). Cerca de $45 \%$ da população reside na área rural, e as principais atividades econômicas da cidade são o turismo e a agropecuária, com destaque para suínos, bovinos, laticínios, milho, soja, arroz e fumo. A produção de fumo no ano de 2010 foi a segunda maior do estado, com 14.875 toneladas cultivadas em 8.500 hectares de terra. Característica marcante dessa população é a colonização da região, no século XIX, por trabalhadores rurais da Pomerânia (região situada no norte da Alemanha e da Polônia) e o predomínio das religiões Luterana e Católica.

Fumicultores devem emitir, anualmente, notas fiscais da venda do tabaco para assegurar seus direitos previdenciários como trabalhadores rurais. De um total de 3.852 notas fiscais emitidas no ano de 2009, foram selecionadas aleatoriamente 1.100, o que garantiu a representatividade da amostra. Todos os indivíduos com 18 anos ou mais de idade que trabalhavam com tabaco pelo menos 15 horas por semana e que cultivaram fumo em 2010 foram elegíveis para o estudo. Notas fiscais selecionadas que foram emitidas por proprietários residentes em outros municípios ou na área urbana de São Lourenço do Sul ou que não cultivavam mais tabaco foram substituídas pela propriedade produtora de fumo mais próxima.

Considerando um nível de 95\% de significância, com uma razão não exposto:exposto de 0,1 , um percentual de tabagismo em não expostos de $20 \%$ e a população estudada de 1.464 indivíduos homens, observou-se que o estudo tem poder estatístico de $80 \%$ para estimar riscos de 1,8 para as associações em estudo.

O estudo ocorreu num contexto de agricultura familiar e foram utilizados dois questionários, um coletou informações sobre a propriedade e outro sobre os indivíduos. O questionário sobre 
a propriedade abordou questões econômicas, como quantidade de tabaco produzida no ano anterior, meios de transporte, maquinário agrícola, diversificação de culturas e criação de animais. O questionário individual foi administrado a todos os indivíduos elegíveis e abordou fatores sociodemográficos (sexo, idade, escolaridade), comportamentais (álcool, tabagismo e hábitos religiosos), exposições ocupacionais e comorbidades. Foi avaliado o consumo de qualquer tipo de cigarro, sendo considerado fumante aquele que consumia um ou mais cigarros por dia há pelo menos um mês, ex-fumante aquele que parou de fumar há mais de um mês e não fumante o que nunca fumou. O consumo de álcool entre homens foi classificado conforme o número de doses diárias ingeridas, sendo considerado bebedor pesado aquele que relatou o consumo de 4 ou mais doses 12. O comportamento religioso foi definido pela participação em atividades religiosas.

Foram avaliadas atividades ocupacionais relacionadas ao cultivo do fumo como plantio, colheita, preparar varas com folhas de fumo para serem alocadas dentro das estufas, subir nos andaimes das estufas, atar as folhas de fumo em pequenos feixes, controlar a temperatura das estufas, enfardamento, entre outras. As cargas de trabalho estudadas foram o relato de trabalho intenso, o tempo (anos) de trabalho com o fumo e de exposição aos agrotóxicos.

As entrevistas foram realizadas no domicílio, após consentimento, por entrevistadores previamente treinados usando personal digital assistants (PDA's). Os entrevistadores eram moradores da região, em sua maioria agentes comunitários de saúde (ACS) e recenseadores do censo 2010, desenvolvido pelo Instituto Brasileiro de Geografia e Estatística (IBGE). O controle de qualidade foi realizado mediante aplicação de uma versão resumida do questionário a $10 \%$ dos respondentes.

Para as análises, o desfecho foi dicotomizado em não fumantes e fumantes/ex-fumantes. Os dados foram analisados conforme as variáveis independentes, com estratificação por sexo, usando o teste de Wald para heterogeneidade e tendência linear. As análises bruta e ajustada utilizaram a regressão de Poisson com variância robusta, "seleção para trás", e foram realizadas apenas em homens, devido ao pequeno número de mulheres tabagistas. A análise multivariada seguiu um modelo hierárquico que incluiu as variáveis sociodemográficas e econômicas no primeiro nível, consumo de álcool e atividades religiosas no segundo e as cargas de trabalho no terceiro nível. As variáveis com valor de $\mathrm{p} \leq 0,20$ foram mantidas no modelo e aquelas com valor de $\mathrm{p}<0,05$ foram consideradas associadas.
O presente estudo foi revisado e aprovado pelo Comitê de Ética da Universidade Federal de Pelotas (ofício número 11/10, registro 40600038), todos os participantes voluntariamente assinaram um termo de consentimento e pessoas detectadas com problemas de saúde foram encaminhadas à assistência médica gratuita.

\section{Resultados}

O estudo entrevistou 2.469 fumicultores (59,3\% homens), moradores de 912 propriedades, após uma taxa de perdas e recusas de $6 \%$ para o sexo masculino.

O trabalho na fumicultura era desempenhado predominantemente por membros da família proprietária, apenas 5,4\% eram empregados ou arrendatários. O número médio de trabalhadores por propriedade foi de 2,7 .

Quase a metade dos trabalhadores apresentava idade entre 25 e 44 anos e $44 \%$ possuíam de zero a quatro anos de estudo. Mais de $95 \%$ dos homens participavam de atividades religiosas. Mais de um terço dos homens trabalhava com tabaco havia pelo menos 20 anos, $27,1 \%$ criavam pelo menos uma espécie animal e $44 \%$ moravam em propriedades que produziam entre 5 e 10 toneladas de tabaco em folha. A exposição a pesticidas por mais de 20 anos foi referida por $43,1 \%$ dos trabalhadores. A grande maioria $(91,5 \%)$ trabalhava mais de 8 horas por dia durante a safra e mais de um terço referiu trabalhar intensamente pelo menos 4 meses do ano. A prevalência de tabagismo foi discrepante entre os sexos, $31,2 \%$ de homens e $3,1 \%$ de mulheres eram fumantes, $19 \%$ dos homens e $4,4 \%$ das mulheres eram ex-fumantes. Consumo pesado de álcool (quatro ou mais doses em uma ocasião) foi referido por $21 \%$ dos trabalhadores homens (Tabelas 1 e 2).

A análise ajustada foi realizada apenas para os homens e mostrou que a jornada de trabalho na safra não esteve associada com o desfecho. Idade esteve diretamente associada com tabagismo, homens com mais de 55 anos apresentaram o maior risco (RP: 2,44). A escolaridade perdeu significância na análise multivariada. A quantidade de tabaco produzida (RP: 0,82) e a criação de animais (RP: 0,66) mostraram-se inversamente associados com tabagismo. $\mathrm{O}$ etilismo pesado foi fator de risco para tabagismo (RP: 1,36) enquanto participar de atividades religiosas foi fator de proteção (RP: 0,69). Homens que trabalhavam intensamente de um a quatro meses no ano fumavam menos (RP: 0,83) do que aqueles que não trabalhavam intensamente. Quanto maior o tempo de trabalho com fumo (RP: 1,17) e de 
Tabela 1

Prevalência de tabagismo e caracterização demográfica, socioeconômica, comportamental e ocupacional dos fumicultores homens. Brasil, 2011.

\begin{tabular}{|c|c|c|c|c|c|}
\hline \multirow[t]{2}{*}{ Variáveis } & \multicolumn{2}{|c|}{ Homens $(N=1.464)$} & \multicolumn{2}{|c|}{ Tabagismo (\%) } & \multirow[t]{2}{*}{ Valor de $p$ * } \\
\hline & $\mathbf{n}$ & \% (IC95\%) & Fumante & Ex-fumante & \\
\hline Idade (anos) & & & & & 0,000 \\
\hline $18-24$ & 205 & $14,0(12,2-15,8)$ & 19,0 & 9,8 & \\
\hline $25-34$ & 383 & $26,2(23,9-28,4)$ & 23,8 & 13,6 & \\
\hline $35-44$ & 319 & $21,8(19,7-23,9)$ & 34,8 & 18,2 & \\
\hline $45-54$ & 317 & $21,6(19,5-23,8)$ & 38,2 & 25,2 & \\
\hline$\geq 55$ & 240 & $16,4(14,5-18,3)$ & 39,6 & 28,3 & \\
\hline Escolaridade (anos) & & & & & 0,000 \\
\hline $0-4$ & 644 & $44,0(41,4-46,5)$ & 37,7 & 21,7 & \\
\hline $5-8$ & 732 & $50,0(47,4-52,6)$ & 25,4 & 17,2 & \\
\hline$\geq 9$ & 88 & $6,0(4,8-7,2)$ & 32,2 & 13,8 & \\
\hline Quantidade de tabaco produzida $(\mathrm{kg})$ & & & & & 0,006 \\
\hline $1-2.500$ & 91 & $6,3(5,0-7,5)$ & 38,9 & 20,0 & \\
\hline $2.501-5.000$ & 396 & $27,2(24,9-29,5)$ & 36,1 & 21,0 & \\
\hline $5.001-10.000$ & 368 & $43,8(41,3-46,4)$ & 29,1 & 19,0 & \\
\hline $10.001-36.000$ & 330 & $22,7(20,5-24,8)$ & 27,2 & 16,3 & \\
\hline Animais (tipos) & & & & & 0,000 \\
\hline Nenhum & 1.060 & $72,9(70,7-75,2)$ & 33,4 & 20,3 & \\
\hline 1 & 258 & $17,8(15,8-19,7)$ & 24,8 & 18,6 & \\
\hline 2 ou mais & 135 & $9,3(7,8-10,8)$ & 25,9 & 9,6 & \\
\hline Relação de trabalho & & & & & 0,027 \\
\hline Família proprietária & 1.385 & $94,6(93,4-95,8)$ & 30,8 & 18,6 & \\
\hline Empregado/Arrendatário & 79 & $5,4(4,2-6,5)$ & 38,0 & 26,6 & \\
\hline Consumo de álcool (doses) & & & & & 0,008 \\
\hline Não bebe & 290 & $19,8(17,7-21,9)$ & 30,0 & 17,9 & \\
\hline$\leq 3$ & 868 & $59,4(56,8-61,9)$ & 28,9 & 18,9 & \\
\hline$\geq 4$ & 304 & $20,8(18,7-22,9)$ & 39,1 & 19,7 & \\
\hline Participa de religião & & & & & 0,000 \\
\hline Não & 54 & $3,8(2,8-4,8)$ & 44,4 & 12,9 & \\
\hline Às vezes & 970 & $68,0(65,5-70,4)$ & 32,7 & 21,4 & \\
\hline Com frequência & 403 & $28,2(25,9-30,6)$ & 25,8 & 14,6 & \\
\hline Tempo que trabalha com tabaco (anos) & & & & & 0,000 \\
\hline$\leq 9$ & 457 & $31,2(28,8-33,6)$ & 29,1 & 12,5 & \\
\hline $10-19$ & 455 & $31,1(28,7-33,5)$ & 27,0 & 18,0 & \\
\hline$\geq 20$ & 551 & $37,7(35,5-40,1)$ & 26,3 & 25,2 & \\
\hline Tempo de exposição aos pesticidas na & & & & & 0,000 \\
\hline \multicolumn{6}{|l|}{ vida (anos) } \\
\hline Sem exposição & 100 & $6,9(5,6-8,2)$ & 31,0 & 13,0 & \\
\hline $1-10$ & 362 & $24,8(22,6-27,1)$ & 24,0 & 12,1 & \\
\hline $11-20$ & 367 & $25,2(22,9-27,4)$ & 27,0 & 18,0 & \\
\hline $21+$ & 628 & $43,1(40,5-45,6)$ & 38,1 & 24,2 & \\
\hline Horas de trabalho durante a safra & & & & & 0,526 \\
\hline$\leq 8$ & 124 & $8,5(7,1-9,9)$ & 32,3 & 14,5 & \\
\hline $9-12$ & 805 & $55,2(52,6-57,7)$ & 31,9 & 19,9 & \\
\hline $13-18$ & 530 & $36,3(33,9-38,8)$ & 29,8 & 18,7 & \\
\hline Meses por ano de trabalho intenso & & & & & 0,003 \\
\hline Nenhum & 181 & $12,4(10,7-14,1)$ & 37,0 & 22,1 & \\
\hline $1-4$ & 709 & $48,7(46,1-51,2)$ & 27,1 & 19,5 & \\
\hline $5-8$ & 479 & $32,9(30,5-35,3)$ & 32,4 & 17,5 & \\
\hline $9+$ & 88 & $6,0(4,8-7,3)$ & 44,3 & 18,2 & \\
\hline
\end{tabular}

IC95\%: intervalo de 95\% de confiança.

* Teste exato de Fisher para a diferença entre as categorias. 
Prevalência de tabagismo por sexo em fumicultores de São Lourenço do Sul, Rio Grande do Sul, Brasil, 2011.

\begin{tabular}{|c|c|c|c|c|c|c|}
\hline & \multicolumn{2}{|c|}{ Homens } & \multicolumn{2}{|c|}{ Mulheres } & \multicolumn{2}{|r|}{ Total } \\
\hline & $\mathrm{n}$ & \% (IC95\%) & $\mathbf{n}$ & \% (IC95\%) & $\mathrm{n}$ & \% (IC95\%) \\
\hline Não fumantes & 729 & $48,9(47,2-53,3)$ & 930 & $92,5(90,9-94,2)$ & 1,659 & $67,2(65,4-69,1)$ \\
\hline Ex-fumantes & 278 & $19,0(17,0-21,0)$ & 44 & $4,4(3,1-5,6)$ & 322 & $13,0(11,7-14,3)$ \\
\hline Fumantes & 457 & $31,2(28,8-33,6)$ & 31 & $3,1(2,0-4,1)$ & 488 & $19,8(18,2-21,3)$ \\
\hline
\end{tabular}

IC95\%: intervalo de 95\% de confiança.

exposição aos pesticidas (RP: 1,22), maior o risco de fumar (Tabela 3).

\section{Discussão}

A alarmante prevalência de tabagismo observada entre os homens fumicultores $(31,2 \%)$ está acima da prevalência nacional de $22 \%$ para o mesmo sexo em área rural 13. Conforme os dados da PNAD 2008, a prevalência de tabagismo entre agricultores é alta (21,9\%) quando comparada com outras ocupações. Os autores ainda ressaltam que o efeito da ocupação persiste mesmo após o ajuste para idade, sexo e renda, contrariando a ideia de outros estudos de que ocupação é apenas um marcador de nível socioeconômico 8,9,10.

A alta prevalência de tabagismo entre homens fumicultores sugere que o trabalho direto com o tabaco estimula o consumo do produto, para além do que acontece com os demais agricultores. Estudos recentes explicam o papel da transmissão familiar e epigenética no uso e dependência de substâncias e na manutenção de comportamentos específicos através das gerações 14,15,16. Dessa forma, fumicultores estariam expostos à nicotina desde a vida embrionária, receberiam a herança epigenética de seus progenitores expostos à nicotina e cresceriam em um cluster familiar de adicção ao tabaco e ao álcool. Sugere-se também que os trabalhadores estariam expostos a elevadas taxas de nicotina durante os meses da safra e poderiam sofrer abstinência no período entressafras. O tabagismo pode ser utilizado para amenizar os sintomas da abstinência e da doença da folha verde, já que tabagistas desenvolvem tolerância à nicotina 17 . Ademais, a rotina diária de manuseio da planta pode levar os fumicultores a naturalizar o risco inerente ao tabaco.

Nesse contexto, chama a atenção a baixa prevalência de tabagismo no sexo feminino. Esse achado é consistente com estudo realizado na
Índia que também apresentou baixas prevalências de tabagismo em mulheres $(0,4 \%) 11$. Acredita-se que fatores culturais, característicos da população pomerana, justifiquem a baixa prevalência de tabagismo em mulheres quando comparada aos homens. A associação direta entre idade e tabagismo pode significar uma redução da prevalência de tabagismo ao longo do tempo, o que estaria em consonância com os inquéritos nacionais 13,18 , ou o início tardio do tabagismo. É importante ter em conta que, embora os mais jovens fumem menos, a prevalência de tabagismo entre aqueles com 35 a 44 anos permanece alta (35\%). A associação inversa entre volume de fumo produzido e produção de animais com tabagismo é consistente com outros estudos que apontam que trabalhadores de nível econômico mais baixo têm maior risco de fumar. O mesmo pode ser verificado entre empregados e arrendatários em relação aos proprietários 3,18,19. A falta de associação entre escolaridade e tabagismo na análise multivariada pode ter ocorrido pelo fato de escolaridade ser um forte marcador de idade no contexto rural.

A associação entre consumo de álcool e tabaco está bem descrita na literatura, inclusive com uma relação dose-resposta monotônica entre as duas substâncias 20. Estudos recentes sugerem que o álcool e a nicotina teriam efeitos farmacológicos interativos que motivam seu uso combinado, além de um papel de reforço e tolerância cruzada no desenvolvimento do consumo, manutenção e dependência de ambas as substâncias 21. Já a associação inversa entre religião e tabagismo existe na literatura há muitas décadas, sendo descrita tanto para adolescentes, quanto adultos e idosos 22,23,24. Estudos apontam a prática religiosa como um amenizadora (buffer) dos efeitos deletérios do estresse sobre a saúde 25 e o estresse como um fator de risco para o início e a manutenção do tabagismo 26,27. Entretanto, ainda há a possibilidade de causalidade reversa caso tabagistas não 
Tabela 3

Análise multivariada: tabagismo e fatores associados entre homens. Brasil, 2011.

\begin{tabular}{|c|c|c|c|c|c|c|}
\hline \multirow[t]{2}{*}{ Variáveis } & \multicolumn{3}{|c|}{ Bruta } & \multicolumn{3}{|c|}{ Ajustada } \\
\hline & RP & IC95\% & Valor de $p$ & RP & IC95\% & Valor de $p$ \\
\hline Idade (anos) & & & 0,000 * & & & 0,000 * \\
\hline $18-24$ & 1,00 & - & & 1,00 & - & \\
\hline $25-34$ & 1,30 & $1,00-1,67$ & & 1,32 & $1,02-1,71$ & \\
\hline $35-44$ & 1,84 & $1,44-2,35$ & & 1,92 & $1,49-1,47$ & \\
\hline $45-54$ & 2,20 & $1,75-2,77$ & & 2,29 & $1,79-2,92$ & \\
\hline$\geq 55$ & 2,36 & $1,86-2,99$ & & 2,44 & $1,89-3,14$ & \\
\hline Escolaridade (anos) & & & $0,000 \star \star$ & & & $0,117 \star \star$ \\
\hline $0-4$ & 1,00 & - & & 1,00 & - & \\
\hline $5-8$ & 1,29 & $1,02-1,64$ & & 0,84 & $0,66-1,08$ & \\
\hline$\geq 9$ & 0,93 & $0,72-1,18$ & & 0,79 & $0,62-1,00$ & \\
\hline Quantidade de tabaco produzida (kg) & & & 0,000 * & & & 0,036 * \\
\hline $1-2.500$ & 1,00 & - & & 1,00 & - & \\
\hline $2.501-5.000$ & 0,97 & $0,79-1,18$ & & 0,95 & $0,77-1,16$ & \\
\hline $5.001-10.000$ & 0,82 & $0,67-0,99$ & & 0,86 & $0,71-1,05$ & \\
\hline $10.001-36.000$ & 0,74 & $0,59-0,93$ & & 0,82 & $0,66-1,03$ & \\
\hline Animais (tipos) & & & 0,000 * & & & 0,000 * \\
\hline Nenhum & 1,00 & - & & 1,00 & - & \\
\hline 1 & 0,81 & $0,68-0,96$ & & 0,82 & $0,70-0,97$ & \\
\hline 2 ou mais & 0,66 & $0,52-0,85$ & & 0,66 & $0,51-0,84$ & \\
\hline Relação de trabalho & & & $0,002 * \star$ & & & $0,000 * \star$ \\
\hline Família proprietária & 1,00 & - & & 1,00 & - & \\
\hline Empregados/Arrendatários & 1,31 & $1,10-1,55$ & & 1,38 & $1,15-1,64$ & \\
\hline Consumo de álcool (doses) & & & 0,002 ** & & & $0,000 * *$ \\
\hline Não bebe & 1,00 & - & & 1,00 & - & \\
\hline$<4$ & 1,00 & $0,86-1,15$ & & 1,06 & $0,92-1,22$ & \\
\hline$\geq 4$ & 1,23 & $1,04-1,44$ & & 1,36 & $1,16-1,59$ & \\
\hline Participa de religião & & & 0,000 * & & & 0,000 * \\
\hline Não & 1,00 & - & & 1,00 & - & \\
\hline Às vezes & 0,94 & - & & 0,93 & $0,72-1,21$ & \\
\hline Com frequência & 0,70 & $0,70-0,88$ & & 0,69 & $0,52-0,91$ & \\
\hline Tempo que trabalha com tabaco (anos) & & & 0,000 * & & & 0,041 * \\
\hline$\leq 9$ & 1,00 & - & & 1,00 & - & \\
\hline $10-19$ & 1,08 & $0,92-1,27$ & & 1,05 & $0,90-1,22$ & \\
\hline$\geq 20$ & 1,48 & $1,29-1,70$ & & 1,17 & $1,00-1,36$ & \\
\hline Tempo de exposição aos pesticidas na vida (anos) & & & 0,000 * & & & 0,039 * \\
\hline Sem exposição & 1,00 & - & & 1,00 & - & \\
\hline $1-10$ & 0,82 & $0,64-1,06$ & & 1,07 & $0,82-1,39$ & \\
\hline $11-20$ & 1,02 & $0,80-1,30$ & & 1,12 & $0,88-1,41$ & \\
\hline$\geq 21$ & 1,41 & $1,13-1,77$ & & 1,22 & $0,98-1,50$ & \\
\hline Meses por ano de trabalho intenso & & & $0,002 \star \star$ & & & $0,018 * \star$ \\
\hline Nenhum & 1,00 & - & & 1,00 & - & \\
\hline $1-4$ & 0,78 & $0,68-0,91$ & & 0,83 & $0,72-0,96$ & \\
\hline $5-8$ & 0,84 & $0,72-0,99$ & & 0,87 & $0,75-1,01$ & \\
\hline $9+$ & 1,06 & $0,85-1,31$ & & 1,06 & $0,85-1,32$ & \\
\hline
\end{tabular}

IC95\%: intervalo de 95\% de confiança; RP: razão de prevalência.

* Teste de Wald para tendência linear;

** Teste de Wald para heterogeneidade. 
se envolvam em práticas religiosas por temerem sofrer algum preconceito.

A associação entre tempo de trabalho na fumicultura e tabagismo pode estar relacionada à naturalização de comportamentos de risco ao longo do tempo, ao aprendizado de estratégias para combater os sintomas de abstinência e da doença da folha verde ou, ainda, ser um efeito residual de idade. Além disso, pode refletir um acúmulo de estresse, tanto por questões típicas de atividades agrícolas como problemas climáticos, migração de familiares, quanto por aspectos relacionados ao sistema integrado de produção, que garante a comercialização do produto, mas muitas vezes deixa o produtor insatisfeito com o preço praticado pela indústria, podendo inclusive provocar a contração de dívidas 28 .

A exposição aos pesticidas ao longo da vida pode reduzir a percepção de vulnerabilidade individual favorecendo a exposição a outros fatores de risco, como tabagismo e etilismo. Isso é consistente com a literatura, que aponta a coocorrência de múltiplos fatores de risco 29,30 e identifica, em um estudo com adolescentes, o aumento da probabilidade da coocorrência com a idade 31 .

Estudo recente mostrou que longas jornadas de trabalho estão associadas com um maior consumo de cigarros e dificuldade de abandono do hábito ${ }^{32}$. Entre os fumicultores, não houve variação na prevalência de fumo com diferentes jornadas de trabalho. Porém, o maior risco de fumar entre quem não trabalha intensamente durante o ano pode refletir maior disponibilidade de tempo para fumar do que os que trabalham intensamente durante a safra.

O desfecho deste estudo considera tabagismo atual o consumo de qualquer cigarro diaria- mente, enquanto censos nacionais brasileiros que avaliam tabagismo em área rural definem como consumo de qualquer tipo de cigarro, diária ou ocasionalmente. Apesar de este estudo utilizar um critério mais restritivo, identificou uma prevalência de tabagismo mais alta. Ao tornar o desfecho dicotômico para a análise multivariada classificou-se ex-fumantes junto com fumantes, por conta do interesse em se detectar os fatores de risco para o fumo independentemente da cessação do hábito. A variabilidade de definições para tabagismo encontrada na literatura dificulta a comparação dos estudos. O presente estudo não coletou informações sobre a forma de tabaco consumida, classificando todos como tabagistas. Os entrevistadores pertenciam a mesma comunidade dos entrevistados; essa característica pode ter subestimado a prevalência de consumo de bebida alcoólica. Cabe ainda considerar a possibilidade de causalidade reversa, comum no delineamento transversal.

O presente estudo aponta a magnitude do tabagismo na população de fumicultores e a associação entre consumo excessivo de álcool, exposição aos agrotóxicos e à nicotina ao longo da vida com tabagismo. Assim, considerando a semelhança no processo de mudança de diferentes comportamentos de risco para o sucesso do Art. 14 da CQCT sobre medidas de redução de demanda relativas à dependência e ao abandono do tabaco na população de fumicultores é importante que a abordagem de tais fatores de risco ocorra de forma concomitante, ampliando a eficiência das ações. Futuros estudos devem avaliar a carga de tabaco, bem como detalhar o papel da epigenética e da absorção dérmica de nicotina no início do hábito de fumar.

\section{Colaboradores}

Todos os autores participaram da concepção do projeto, análise e interpretação dos dados, redação e aprovação da versão final e são responsáveis por todos os aspectos do trabalho, garantindo a exatidão e integridade de qualquer parte da obra.

\section{Agradecimentos}

Agradecemos à Secretaria de Saúde de São Lourenço do Sul pelo apoio durante todo o trabalho de campo e aos fumicultores que disponibilizaram seu precioso tempo participando do estudo. À FAPERGS e ao CNPq.

\section{Referências}

1. Instituto Nacional de Câncer José Alencar Gomes da Silva. Programa Nacional de Controle do Tabagismo. http://www2.inca.gov.br/wps/wcm/ connect/acoes_programas/site/home/nobrasil/ programa-nacional-controle-tabagismo (acessado em Mai/2015).

2. Palipudi KM, Gupta PC, Sinha DN, Andes LJ, Asma S, McAfee T. Social determinants of health and tobacco use in thirteen low and middle income countries: evidence from Global Adult Tobacco Survey. PLoS One 2012; 7:e33466. 
3. World Health Organization. WHO report on the global tobacco epidemic, 2013: enforcing bans on tobacco advertising, promotion and sponsorship. http://www.who.int/tobacco/global_report/2013/ en/ (acessado em Mai/2015).

4. World Health Organization. WHO global report on trends in prevalence of tobacco smoking 2015. http://apps.who.int/iris/bitstream/10665/ 156262/1/9789241564922_eng.pdf (acessado em Mai/2015).

5. Bilano V, Gilmour S, Moffiet T, d'Espaignet ET, Stevens GA, Commar A, et al. Global trends and projections for tobacco use, 1990-2025: an analysis of smoking indicators from the WHO Comprehensive Information Systems for Tobacco Control. Lancet 2015; 385:966-76.

6. Ng M, Freeman MK, Fleming TD, Robinson M, Dwyer-Lindgren L, Thomson B, et al. Smoking prevalence and cigarette consumption in 187 countries, 1980-2012. JAMA 2014; 311:183-92.

7. Harper S, McKinnon B. Global socioeconomic inequalities in tobacco use: internationally comparable estimates from the World Health Surveys. Cancer Causes Control 2012; 23 Suppl 1:11-25.

8. Lee DJ, Fleming LE, Arheart KL, LeBlanc WG, Caban AJ, Chung-Bridges $\mathrm{K}$, et al. Smoking rate trends in U.S. occupational groups: the 1987 to 2004 National Health Interview Survey. J Occup Environ Med 2007; 49:75-81.

9. Smith DR. Tobacco smoking by occupation in Australia and the United States: a review of national surveys conducted between 1970 and 2005. Ind Health 2008; 46:77-89.

10. Barros AJ, Cascaes AM, Wehrmeister FC, MartinezMesa J, Menezes AM. Tobacco smoking in Brazil: regional inequalities and prevalence according to occupational characteristics. Ciênc Saúde Coletiva 2011; 16:3707-16.

11. Parikh JR, Gokani VN, Doctor PB, Kulkarni PK, Shah AR, Saiyed HN. Acute and chronic health effects due to green tobacco exposure in agricultural workers. Am J Ind Med 2005; 47:494-9.

12. U.S. Department of Agriculture; U.S. Department of Health and Human Service. Dietary guidelines for Americans, 2010. Washington DC: U.S. Government Printing Office; 2010.

13. Instituto Brasileiro de Geografia e Estatística. Pesquisa Nacional de Saúde, 2013. http://www.ibge. gov.br/home/estatistica/populacao/pns/2013/ (acessado em Mai/2015)

14. Bierut LJ, Dinwiddie SH, Begleiter H, Crowe RR, Hesselbrock V, Nurnberger Jr. JI, et al. Familial transmission of substance dependence: alcohol, marijuana, cocaine, and habitual smoking: a report from the Collaborative Study on the Genetics of Alcoholism. Arch Gen Psychiatry 1998; 55:982-8.

15. Taki FA, Pan X, Lee MH, Zhang B. Nicotine exposure and transgenerational impact: a prospective study on small regulatory microRNAs. Sci Rep 2014; 4:7513.

16. Vassoler FM, Sadri-Vakili G. Mechanisms of transgenerational inheritance of addictive-like behaviors. Neuroscience 2014; 264:198-206.
17. Fassa AG, Faria NM, Meucci RD, Fiori NS, Miranda VI, Facchini LA. Green tobacco sickness among tobacco farmers in southern Brazil. Am J Ind Med 2014; 57:726-35.

18. Instituto Nacional de Câncer; Organização Pan-Americana da Saúde. Pesquisa Especial de Tabagismo - PETab: relatório Brasil. Rio de Janeiro: Instituto Nacional de Câncer; 2011.

19. Ross H, Chaloupka FJ. Economic policies for tobacco control in developing countries. Salud Pública Méx 2006; 48 Suppl 1:S113-20.

20. Falk DE, Yi HY, Hiller-Sturmhofel S. An epidemiologic analysis of co-occurring alcohol and tobacco use and disorders: findings from the National Epidemiologic Survey on Alcohol and Related Conditions. Alcohol Res Health 2006; 29:162-71.

21. Oliver JA, Blank MD, van Rensburg KJ, MacQueen DA, Brandon TH, Drobes DJ. Nicotine interactions with low-dose alcohol: pharmacological influences on smoking and drinking motivation. J Abnorm Psychol 2013; 122:1154-65.

22. Koenig HG, George LK, Cohen HJ, Hays JC, Larson DB, Blazer DG. The relationship between religious activities and cigarette smoking in older adults. J Gerontol A Biol Sci Med Sci 1998; 53:M426-34.

23. Lucchetti G, Peres MF, Lucchetti AL, Koenig HG. Religiosity and tobacco and alcohol use in a Brazilian shantytown. Subst Use Misuse 2012; 47:837-46.

24. Whooley MA, Boyd AL, Gardin JM, Williams DR. Religious involvement and cigarette smoking in young adults: the CARDIA study (Coronary Artery Risk Development in Young Adults) study. Arch Intern Med 2002; 162:1604-10.

25. Williams DR, Larson DB, Buckler RE, Heckmann RC, Pyle CM. Religion and psychological distress in a community sample. Soc Sci Med 1991; 32:1257-62.

26. Cui X, Rockett IR, Yang T, Cao R. Work stress, life stress, and smoking among rural-urban migrant workers in China. BMC Public Health 2012; 12:979.

27. Lindstrom M, Moden B, Rosvall M. A life-course perspective on economic stress and tobacco smoking: a population-based study. Addiction 2013; 108:1305-14.

28. Vogt OP. A produção do fumo em Santa Cruz do Sul, RS: 1894-1993. Santa Cruz do Sul: Edunisc; 1997.

29. Noar SM, Chabot M, Zimmerman RS. Applying health behavior theory to multiple behavior change: considerations and approaches. Prev Med 2008; 46:275-80.

30. Prochaska JJ, Spring B, Nigg CR. Multiple health behavior change research: an introduction and overview. Prev Med 2008; 46:181-8.

31. Driskell MM, Dyment S, Mauriello L, Castle P, Sherman K. Relationships among multiple behaviors for childhood and adolescent obesity prevention. Prev Med 2008; 46:209-15.

32. Angrave D, Charlwood A, Wooden M. Working time and cigarette smoking: evidence from Australia and the United Kingdom. Soc Sci Med 2014; 112:72-9. 


\section{Abstract}

This study aimed to describe the prevalence of smoking and associated factors in a cross-sectional population-based sample of 2,464 tobacco farmers in 2011. Multivariate analysis in men assessed the association between smoking and socioeconomic, behavioral, and occupational variables. Some $31.2 \%$ of men and $3.1 \%$ of women were current smokers. In men, smoking was directly associated with age, schooling, income, heavy drinking, time at work in tobacco farming, and time of exposure to pesticides. Employment relationship was a risk factor for smoking, and participation in religious activities was a protective factor. Male tobacco farmers showed multiple risk behaviors and higher smoking prevalence than other farmers. Ignoring the risk and cultural legacy may be common factors for these behaviors and suggest combined approaches.

Smoking; Tobacco; Agriculture; Rural Population

\section{Resumen}

El objetivo fue describir la prevalencia de tabaquismo $y$ factores asociados en cultivadores de tabaco. Estudio transversal de base poblacional, con 2.464 cultivadores de tabaco, en 2011. Muestra obtenida por aleatorización de las facturas emitidas por los trabajadores. El análisis multivariado en hombres evaluó la asociación entre tabaquismo y variables socioeconómicas, comportamentales y ocupacionales. Un 31,2\% de los hombres y 3,1\% de las mujeres eran tabaquistas actuales. Entre hombres, el tabaquismo se asoció directamente con la edad, escolaridad, renta, ser bebedor habitual, tiempo de trabajo en el cultivo de tabaco y tiempo de exposición a pesticidas. Las relaciones de trabajo fueron un factor de riesgo para el tabaquismo y participar en actividades religiosas un factor de protección. Los cultivadores de tabaco hombres presentaron múltiples comportamientos de riesgo y una prevalencia de tabaquismo mayor que otros agricultores. La naturalización del riesgo y la herencia cultural pueden ser factores comunes a estos comportamientos y sugieren enfoques conjuntos.

Hábito de Fumar; Tabaco; Agricultura; Población Rural
Recebido em 29/Jul/2015

Versão final reapresentada em 01/Out/2015

Aprovado em 22/Fev/2016 